\title{
A Phenomenological Estimate of the Binding Energy of Heavy Dimesons *
}

\author{
D. Janc ${ }^{\dagger}$ and M. Rosina ${ }^{\ddagger}$ \\ Faculty of Mathematics and Physics, University of Ljubljana, \\ Jadranska 19, P.O. Box 2964, 1001 Ljubljana, Slovenia, \\ and J. Stefan Institute, Ljubljana, Slovenia
}

\begin{abstract}
A phenomenological estimate is derived such that the binding energies of dimesons are expressed as combinations of masses of different mesons and baryons. The estimate is almost model-independent, the only major assumptions being that the wave functions of the two light quarks in $\Lambda_{c}, \Lambda_{b}$ and in the $\bar{c} \bar{c} q q$ and $\bar{b} \bar{b} q q$ dimesons are very similar, and that for heavy quarks the $Q Q$ interaction is half as strong as the $Q \bar{Q}$ interaction. We get $\bar{b} \bar{b} q q(\mathrm{I}=0, \mathrm{~J}=1)$ bound by about $100 \mathrm{MeV}$ and $\bar{c} \bar{c} q q$ unbound.
\end{abstract}

\section{Introduction}

The constituent quark model has been rather successful in describing the properties of individual hadrons [ [4, 2, 迆. The extrapolation to two-hadron systems is, however, still rather uncertain and the predictions of various quark models sometimes differ dramatically. One source of discrepancy is our limited knowledge about the "correct" effective quark-quark interaction, and another source are different choices of model parameters designed to fit

*submitted to Few Body Systems

${ }^{\dagger}$ E-mail: damjan.janc@ijs.si

${ }_{\ddagger}^{\ddagger}$ E-mail: mitja.rosina@ijs.si 
different subsets of hadrons. Even the calculation of the nucleon-nucleon interaction in the model with two three-quark clusters is quite unreliable.

Much can be learned by studying a simpler two-hadron system: two heavy mesons. The lowest states should be very narrow (longlived) since they can decay only weakly. Although their width is only $\sim 10^{-3} \mathrm{eV}$ (corresponding to a lifetime of $\sim 1 \mathrm{ps}$ ) they are difficult to detect because of a low production cross section. Nevertheless, they are interesting theoreticaly, to confront different models. The detailed calculations in the literature [4, 5] rely on particular quark models, therefore we attempt an almost model-independent phenomenological estimate.

In Sect. 2 we present our phenomenological estimate of the binding energy in order to support the expectation that some heavy dimesons may be bound. The estimate is mainly based on the assumption that the wave functions of the two light quarks around the heavy quark in $\Lambda_{c}, \Lambda_{b}$ and around the antidiquark in the $\bar{c} \bar{c} q q$ and $\bar{b} \bar{b} q q$ dimesons are very similar. This assumpton implies that the heavy antidiquark in a colour triplet state acts just like a very heavy quark and that the $1 / m$ corrections are neglected.

This assumption is supported by the experimental observation that the masses of many meson or baryon pairs with $c$ quark replaced by $b$ quark differ by the same amount (essentially by the quark mass difference): $\tilde{B}-\tilde{D}=$ $3341 \mathrm{MeV}, \tilde{B}_{s}-\tilde{D}_{s}=3328 \mathrm{MeV}, \Lambda_{b}-\Lambda_{c}=3340 \mathrm{MeV}$.

The second assumption is that for heavy quarks the $Q Q$ interaction is half as strong as the $Q \bar{Q}$ interaction. This is true for the one-gluon-exchange interacton and popular for the confining interaction. In potential models with 2-body interaction it is difficult to avoid the $\lambda \cdot \lambda$ factor in the interaction without closing all open channels for white clusters. One should note also that for heavy quarks the one-pion-exchange potential does not apply.

In Sect. 3 we show that several refinements may give corrections of \pm 20 $\mathrm{MeV}$ but they do not change the qualitative features and conclusions.

We comment the results in the Conclusion where we explain why some previous model calculations are in agreement with our phenomenological results and why some other calculations which disagree are irrelevant.

\footnotetext{
${ }^{1}$ In this paper we shall denote the masses of particles just by their names, and the tilde denotes a hyperfine average (e.g. $\tilde{D}=\frac{1}{4} D+\frac{3}{4} D^{*}$ ).
} 


\section{The phenomenological relation for the bind- ing energy of dimesons}

The results in this section, Eqs. (5) and (8), are the main message of our paper. In next section we only scrutinize the assumptions and neglected terms and estimate the minor corrections. While in the present section the calculations are very simple, essentially interpolations between experimental hadron masses, the corrections in next section rely strongly on detailed constituent quark model calculations [6].

We derive phenomenological relations between dimesons, mesons and baryons by making two assumptions, (i) the equality of the wavefunction of two light quarks in the dimeson and in a heavy baryon, and (ii) the similarity between the subsystem of two heavy quarks in the dimeson and the quark-antiquark system in a heavy meson.

The first assumption implies that the heavy antidiquark in a colour triplet state acts just like a very heavy quark and that the $1 / m$ corrections are neglected; however, there is no prejudice for the light-light subsystem in favour of the one-gluon-exchange or one-Goldstone-boson-exchange interaction.

The second assumption implies that effective interaction between two heavy quarks in colour triplet state is half as strong as the effective quarkantiquark interaction in colour singlet state; therefore the binding energies of diquarks are weaker than those of mesons, and the distances increase. We do not prejudice the radial dependence of the effective interaction (the flavour-dependent spin-spin interaction is treated later as a perturbation).

We call the $u$ and $d$ quarks $q$ and the dimesons $(\bar{b} \bar{b} q q)=T_{b b},(\bar{c} \bar{c} q q)=T_{c c}$ (the symbol $T$ reminds of the terminology tetraquarks).

The binding energy $E_{b \bar{b}}$ of a quark and antiquark in a meson is a function of the reduced mass only, e.g. $\Upsilon=b+b+E_{b \bar{b}}, E_{b \bar{b}}=F(m=b / 2)$. For the diquark $b b$ the Schrödinger equation is similar as for the $b \bar{b}$ meson with twice weaker interaction

$$
\left[\frac{p^{2}}{2(b / 2)}+V_{b b}\right] \psi=\frac{1}{2}\left[\frac{p^{2}}{2(b / 4)}+V_{b \bar{b}}\right] \psi=E_{b b} \psi
$$

To get the similarity, we have mimicked the kinetic energy with half smaller reduced mass. The binding energy is then $E_{b b}=\frac{1}{2} F(b / 4)$.

Now we compare the following hadrons

$$
T_{b b}=2 b+2 q+E_{b b}+E_{q q Q}
$$




$$
\begin{aligned}
\Upsilon & =2 b+E_{b \bar{b}} \\
\Lambda_{b} & =b+2 q+E_{q q Q}
\end{aligned}
$$

where $E_{q q Q} \approx E_{q q(\bar{b} \bar{b})} \approx E_{q q b}$ is the potential plus kinetic energy contribution of the two light quarks in the field of a "heavy quark". The quantity $E_{q q Q}$ is taken from the experimental data on $\Lambda_{c}$ and $\Lambda_{b}$ and we do not need to calculate it.

We obtain the phenomenological relation

$$
T_{b b}=\Lambda_{b}+\frac{1}{2} \Upsilon+\delta E_{b b}, \quad \delta E_{b b}=\frac{1}{2}[F(b / 4)-F(b / 2)] .
$$

An analogous comparison gives for the charm-charm dimeson

$$
T_{c c}=\Lambda_{c}+\frac{1}{2} J / \psi+\delta E_{c c}, \quad \delta E_{c c}=\frac{1}{2}[F(c / 4)-F(c / 2)] .
$$

The binding of the $(I=0, J=1)$ dimesons is expressed with respect to the corresponding thresholds. In order to be unambiguous we give in Appendix all experimental data and all references to model parameters which we need now or later.

$$
\begin{aligned}
\triangle T_{b b} & =\Lambda_{b}+\frac{1}{2} \Upsilon-B-B^{*}+\delta E_{b b}=-250 \mathrm{MeV}+\delta E_{b b}, \\
\triangle T_{c c} & =\Lambda_{c}+\frac{1}{2} J / \psi-D-D^{*}+\delta E_{c c}=-42 \mathrm{MeV}+\delta E_{c c}
\end{aligned}
$$

Now comes an important idea how to obtain phenomenologically the "corrections" $\delta E$. In Fig.(四) we plot the phenomenological binding energies obtained from experimental meson masses and from a popular sets of quark masses, as a function of the reduced masses of the $b \bar{b}, c \bar{c}, b \bar{s}, c \bar{s}, b \bar{q}$ mesons. We chose the quark masses proposed by Bhaduri et al. $(B D)$ [7] and by SilvestreBrac $(A L 1)$ [2], collected in Appendix; because of similarity between the two sets only the Fig.(11) for the set $(B D)$ is presented. The results are only weakly dependent on quark masses. We then interpolate in order to obtain the values of $F$ for the scaled reduced masses of diquarks. The idea to interpolate between experimental meson masses has been used before [8], but their construction of dimesons is quite different: a light diquark and a heavy antidiquark bound as a meson; however, the assumption of a light diquark is less justified than our construction with full freedom for the two light quarks.

For the interpolation we use the following data

$$
\frac{1}{2} E_{b \bar{b}}=\frac{1}{2} \Upsilon-b \equiv \frac{1}{2} F\left(\frac{1}{2} b\right)=-529 \mathrm{MeV}(B D), \quad-497 \mathrm{MeV}(A L 1),
$$




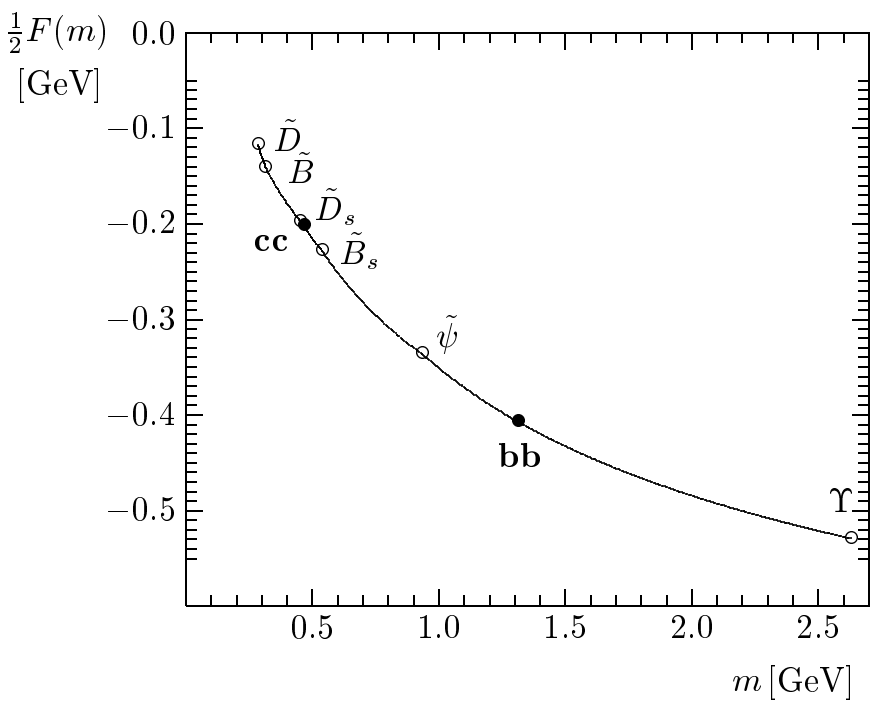

Figure 1: Interpolation for the $b b$ binding energy $-B D$ parameters (The curve for AL1 parameters is similar.)

$$
\begin{aligned}
& \frac{1}{2} E_{c \bar{c}}=\frac{1}{2} \tilde{\psi}-c \equiv \frac{1}{2} F\left(\frac{1}{2} c\right)=-336 \mathrm{MeV}(B D), \quad-302 \mathrm{MeV}(A L 1), \\
& \frac{1}{2} E_{c \bar{s}}=\frac{1}{2}\left(\tilde{D}_{s}-c-s\right) \\
& \equiv \frac{1}{2} F\left(\left(c^{-1}+s^{-1}\right)^{-1}\right)=-197 \mathrm{MeV}(B D), \quad-168 \mathrm{MeV}(A L 1)(6)
\end{aligned}
$$

Here we used the hyperfine averages $\tilde{\psi}=\frac{3}{4} J / \psi+\frac{1}{4} \eta_{c}$ and similarly for $D_{s}$; for $\Upsilon$ we take the $S=1$ state since $\eta_{b}$ is unknown and the splitting is anyway small.

For the interpolation between $\Upsilon$ and $\tilde{\psi}$ we plot in Fig.(1) curves of the form $\sigma m^{-1 / 3}+\tau$. Such interpolation corresponds to the scaling for the linear quark-antiquark potential; it also gives a rather straight line for phenomenological binding energies if plotted against the abscissa $m^{-1 / 3}$. We get

$$
\begin{array}{rlrl}
B D: & \frac{1}{2} F\left(\frac{1}{4} b\right) & =\frac{1}{2} F(1315 \mathrm{MeV})=-407 \mathrm{MeV} . \\
A L 1: & \frac{1}{2} F\left(\frac{1}{4} b\right)=\frac{1}{2} F(1307 \mathrm{MeV})=-375 \mathrm{MeV} .
\end{array}
$$

There is some uncertainty in the interpolation. The extreme choices of linear interpolation of $F$ versus $1 / m$ (or $m$ ) give $15 \mathrm{MeV}$ stronger (27 MeV weaker) binding, respectively, suggesting an error $\pm 15 \mathrm{MeV}$. The binding energy $\triangle T_{b b}$ is then

$$
B D: \quad \delta E_{b b}=+122 \pm 15 \mathrm{MeV}, \quad \triangle T_{b b}=-128 \pm 15 \mathrm{MeV}
$$




$$
A L 1: \quad \delta E_{b b}=+122 \pm 15 \mathrm{MeV}, \quad \triangle T_{b b}=-128 \pm 15 \mathrm{MeV}
$$

These values are very close to the result $\triangle T_{b b}=-131 \mathrm{MeV}$ of a detaild 4-body calculation with $B D$ interaction [四].

For $T_{c c}$ it is even easier to interpolate since the reduced mass $c / 4$ happens to lie very close to the reduced mass of the $\tilde{D}_{s}=c \bar{s}$ meson so that

$$
F\left(\frac{1}{4} c\right)=F(467 \mathrm{MeV}) \approx F\left(\left(c^{-1}+s^{-1}\right)^{-1}\right)=F(454 \mathrm{MeV})
$$

for $B D$ and similar for $A L 1$. However, the choice of the strange quark mass brings some slight additional model-dependence.

We get

$$
\begin{aligned}
B D: & \delta E_{c c}=+139 \mathrm{MeV}, & \triangle T_{c c}=+97 \mathrm{MeV} \\
A L 1: & \delta E_{b b}=+134 \mathrm{MeV}, & \triangle T_{b b}=+92 \mathrm{MeV}
\end{aligned}
$$

Therefore we expect rather reliably that the $D D^{*}$ system $T_{c c}$ is unbound.

It is amusing to perform an analytic calculation of $\delta E$ by mimicking the $\alpha / r+\kappa+\lambda r$ potential by a logarythmic potential $U \ln \left(r / r_{0}\right)$. The ground state energy of mesons is

$$
E=F(m)=U\left(\epsilon-\frac{1}{2} \ln \left[2 m r_{0}^{2} U / \hbar^{2}\right]\right)
$$

where $m$ is the reduced mass and $\epsilon=1.0443$ is obained by the numerical solution of the Schrödinger equation in dimensionless form. Then we obtain for any quark $Q=c, b, \ldots$

$$
\delta E_{Q Q}=\frac{1}{2}[F(Q / 4)-F(Q / 2)]=\frac{\ln 2}{4} U=127 \mathrm{MeV}
$$

which is surprisingly close to the above phenomenological estimates for the $b b$ and $c c$ diquarks. The strength $U=733 \mathrm{MeV}$ is taken from ref. [9].

\section{Refinements}

In this section we make several refinements and corrections in order to test our assumptions and approximations. It turns out that the refinements may change the results only by $10-30 \mathrm{MeV}$ so that our qualitative conclusions remain valid. 


\subsection{Sensitivity to quark masses}

We repeat the calculation with a rather different choice of masses. We explore smaller masses. We propose the following phenomenological choice in which we assume that the wave functions of both partners are the same. Then mass differences of mesons correspond to mass difference of quarks, and the ratio of hyperfine splits corresponds to the mass ratio of quarks.

$$
\begin{aligned}
b-c \approx \tilde{B}-\tilde{D}=3341 \mathrm{MeV}, & b & =4941 \mathrm{MeV} \\
b / c \approx \frac{D^{*}-D}{B^{*}-B}=3.087, & c & =1600 \mathrm{MeV}
\end{aligned}
$$

The results (using the linear interpolation of $E$ versus $m^{-1 / 3}$ ) are

$$
E_{b b}=-128 \mathrm{MeV}, \quad \delta E_{b b}=83 \mathrm{MeV}, \quad \triangle T_{b b}=-167 \mathrm{MeV}
$$

As we see, smaller quark masses yield a somewhat stronger binding. The reason is indirect, the separation of meson masses in quark masses plus binding energy is to some extent arbitrary, smaller quark masses mean less negative binding energy and therefore a smaller $\delta E$.

For the strange quark such suggestions are very uncertain since the assumption of equal wave functions is not justified. Nevertheless, we take the suggestions as a guidance (we take for the light quark $q=320 \mathrm{MeV}$.)

$$
\begin{array}{rlrl}
c-s & \sim \tilde{D}-\tilde{K}=1179 \mathrm{MeV}, & s & \sim 421 \mathrm{MeV} \\
s / c \sim \frac{D^{*}-D}{K^{*}-K}=0.355, & s & \sim 568 \mathrm{MeV} \\
s-q & \sim \tilde{D}_{s}-\tilde{D}=103 \mathrm{MeV}, & s & \sim 420 \mathrm{MeV} \\
s-q & \sim \tilde{B}_{s}-\tilde{B}=90 \mathrm{MeV}, & s & \sim 410 \mathrm{MeV}
\end{array}
$$

and choose for the phenomenological calculation $s=420 \mathrm{MeV}$. Since the reduced mass for the $c c$ diquark $m=c / 4=400 \mathrm{MeV}$ is close to the reduced masses for $c \bar{s}(333 \mathrm{MeV})$ and $b \bar{s}(387 \mathrm{MeV})$ we interpolate linearly and we obtain

$$
E_{c c}=+19 \mathrm{MeV}, \quad \delta E_{c c}=85 \mathrm{MeV}, \quad \triangle T_{c c}=+43 \mathrm{MeV}
$$

The $D D^{*}$ dimeson is unbound also for these smaller masses. We conclude that there is some model-dependence on masses, but not too strong. 


\subsection{The spin-spin interaction}

Since the $b b$ diquark is colour antisymmetric and orbital symetric it must be spin symmetric $(\mathrm{S}=1)$ and is analogous to $\Upsilon$. On the other hand, to get $F(b / 4)$ we had to interpolate between $F(b / 2)$ and $F(c / 2)$. Since the spin-spin interaction is flavour dependent it scales differently with masses as the spinindependent part of interaction and we have interpolated between hyperfine averages. Therefore $F_{S=1}(c / 2)$ corresponding to the $b b$ system should be lifted by $\frac{1}{2} \cdot \frac{1}{4}\left(J / \psi-\eta_{c}\right)(c / b)=5 \mathrm{MeV}$ (for $B D$ ) and $F(b / 4)$ even less, which is negligible. (Since $\eta_{b}$ is still unknown, we have actually used $\Upsilon$ instead of $\tilde{\Upsilon}$ and the lifting is still smaller).

For the $c c$ diquark the shift is sligtly larger. In the hyperfine average, $E_{c c}$ is close to $\frac{1}{2} E_{c \bar{s}}$. The splitting $D_{s}^{*}-D_{s}=144 \mathrm{MeV}$ suggests the splitting between $c c$ states $\frac{1}{2}\left(D_{s}^{*}-D_{s}\right)(s / c)=23 \mathrm{MeV}$. The $S=1$ diquark will then be $6 \mathrm{MeV}$ higher than the hyperfine average and then also the estimate for the $T_{c c}$ energy comes $6 \mathrm{MeV}$ higher. But $T_{c c}$ is anyway unbound by almost $100 \mathrm{MeV}$.

\subsection{The centre-of-mass motion}

In our phenomenological estimation we took the kinetic energy of the relative motion of the light diquark against the heavy diquark in $T_{b b}$ to be equal to the kinetic energy of the light diquark against the $b$ quark in $\Lambda_{b}$. To evaluate the correction to the kinetic energy, we repeated the calculations carried out in [5] where the $B D$ parameter set was used.

For the orbital part of the $T_{b b}$ wave function we took a simple ansatz where the wave functions of heavy diquark, light diquark and their relative motion are Gaussians with widths $b_{i}$ which are chosen so that the the energy of $T_{b b}$ is minimal

$$
\begin{gathered}
R=\exp \left(-\left(r_{1}^{2} / 2 b_{1}^{2}+r_{2}^{2} / 2 b_{2}^{2}+r_{3}^{2} / 2 b_{1}^{3}\right)\right), \\
b_{1}=0.59 \mathrm{fm}, \quad b_{2}=0.23 \mathrm{fm}, \quad b_{3}=0.60 \mathrm{fm} .
\end{gathered}
$$

Here $r_{1}, r_{2}$ and $r_{3}$ are the distances between the heavy quarks, between the light quarks and between the centres-of-masses of the two clusters, respectively. This wave function is symmetric in permutation of the two light quarks and in permutation of the two heavy quarks so that the spin-colour part of the function for $T_{b b}$ in the state with total isospin, spin and parity 
$I S P=10^{+}$must have the form:

$$
S_{q q} C_{q q} S_{\bar{b} \bar{b}} C_{\bar{b} \bar{b}}=(0)_{q q}(\overline{3})_{q q}(1)_{\bar{b} \bar{b}}(3)_{\bar{b} \bar{b}}
$$

Since we expect that this is the most important configuration we neglect the contribution of other configurations in the calculation of the correction to the kinetic energy. We also expect that a more elaborate wave function of the type [5] which gives $\sim 30 \mathrm{MeV}$ smaller mass of $T_{b b}$ would not change this correction by more than a few $\mathrm{MeV}$.

The calculated kinetic energy of relative motion of $(\bar{b} \bar{b})--(q q)$ is 253 $\mathrm{MeV}$. This kinetic energy should be smaller than the corresponding kinetic energy of $b--(q q)$ in $\Lambda_{b}$ used in our estimation by a factor

$$
\frac{1 / 2 q+1 / b}{1 / 2 q+1 / 2 b}=1.06 .
$$

Therefore the mass of $T_{b b}$ should be for $15 \mathrm{MeV}$ smaller than the phenomenologicaly estimated mass.

We also confirmed that the width of the spatial wave function of the heavy diquark in $T_{b b}$ (calculated in the approximation with one Gaussian) is the same as in an isolated system of two $b$ quarks. This supports our assumption that the presence of light quarks in dimeson does not change the binding energy of the heavy diquark (the change is less than $1 \mathrm{MeV}$ ).

\subsection{The finite size of the heavy diquark}

We again assume that the most important configuration in $T_{b b}$ is one with antitriplet colour function for the light diquark and triplet colour function for heavy diquark. This makes $T_{b b}$ very similar to the baryon $\Lambda_{b}$ which we use in our phenomenological estimation. Here we calculate the correction to the heavy-light interaction energy because of the finite size of the heavy diquark. If we make the limit $b_{2} \rightarrow 0$ in the wave function defined in the previous subsection the binding energy is by $18 \mathrm{MeV}$ less negative (for $B D$ parameters, and a similar value for $A L 1$ ). So this correction has the opposite sign as the correction due to the smaller kinetic energy and they almost cancel. 


\subsection{The colour configuration mixing}

In $T_{b b}$ the light diquark and the heavy diquark can also be in colour (anti)sextet state. Such additional configurations are not present in $\Lambda_{b}$ and can lower the energy of $T_{b b}$. This effect was calculated in Born-Oppenheimer approximation since such approximation is very suitable for studing simultaneously the significance of sextet configurations as well as the tendency for two-cluster configurations. (next subsection).

The wave function of $T_{b b}$ is taken as a linear combination of seven configurations $\Phi_{i}$ listed in table 1 (for $\Phi_{i}$ we choose an orthogonalized basis)

$$
\Psi(\mathbf{r})=\sum_{i} c_{i}(\mathbf{r}) \Phi_{i}(\mathbf{r})
$$

We solved accurately seven coupled differential equations

$$
-\frac{\hbar^{2} \nabla^{2}}{2 m_{B B}} c_{i}(\mathbf{r})+\sum_{j} V_{i j}(\mathbf{r}) c_{j}(\mathbf{r})=E c_{i}(\mathbf{r})
$$

where $m_{B B}$ is the reduced mass of the two $B=\bar{b} q$ clusters and the effective Born-Oppenheimer potential $V_{i j}(\mathbf{r})$ contains the kinetic energies of the light quarks as well as all interaction energies. The calculation is done in the spirit of the generator coordinate method (or resonating group method) except that $V_{i j}(\mathbf{r})$ is calculated with fixed heavy quarks.

With the BD parameter set the binding energy is $-70 \mathrm{MeV}$ if all seven configuration from table 1 are used, and $-45 \mathrm{MeV}$ if only the first three configurations (with colour triplet wave functions) are considered. We find it plausible that colour sextet configurations, which were neglected in our phenomenological estimation reduce the mass of $T_{b b}$ by $\sim 25 \mathrm{MeV}$ also in an accurate calculation. The effect is somewhat larger in the case of $T_{c c}$ due to the smaller mass in the denominator of the spin-spin interaction which mixes the configurations, but the energy is still far above the threshold.

\subsection{Search for a two-cluster configuration ("molecule" $\left.B B^{*}\right)$}

At short distance, the colour triplet configurations $\Phi_{3+}, \Phi_{1+}$ and $\Phi_{3-}$ give a Coulomb-like attraction between the two $B=\bar{b} q$ clusters while the colour sextet configurations give repulsion. At intermediate distances, on the other 
hand, one can gain energy with a strong mixing between triplet and sextet configurations. It turns out, however, that in the Born-Oppenheimer wave function of the previous subsection, the amplitudes of colour sextet configurations are more than ten times smaller than the amplitudes of colour triplet configurations. This has the consequence that the maximum probability of the relative motion occurs at the origin. Detailed calculations [6] gave in fact no bound states of $T_{b b}$ with a two-cluster ("molecular" or "covalent") structure.

In Fig. 2 we show the "adiabatic' Born-Oppenheimer potential, where at each distance $\mathbf{r}$ separately the matrix $V_{i j}$ has been diagonalized. This is not an accurate approximation, but it demonstrates well the point that no second minimum appears in the $B B^{*}$ effective potential. Of course, the actual accurate calculations of eigenenergies were performed with the full set of equations (13).

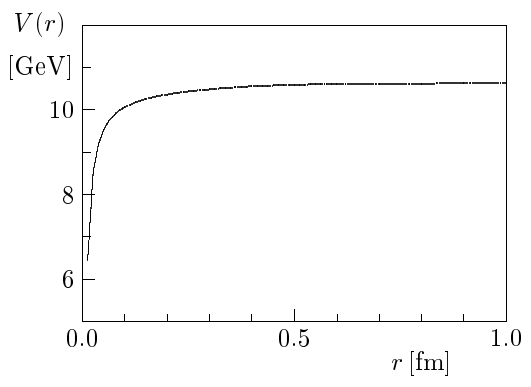

Figure 2: The adiabatic Born-Oppenheimer potential for the $B B^{*}$ system

\section{Conclusion}

Assuming the equality of the wave function of two light quarks in the dimeson and in $\Lambda_{b}$ as well as the similarity between the subsystem of two heavy quarks in the $B B^{*}$ dimeson and the quark-antiquark system in botomium (with scaled interaction strength) we predict a binding of the $T_{b b}=B B^{*}$ dimeson of about $-100 \mathrm{MeV}$. Analogous comparison with $J / \psi$ and $\Lambda_{c}$ predicts the dimeson $T_{c c}=D D^{*}$ to be unbound.

The binding energy of the $B B^{*}$ dimeson of about $-100 \mathrm{MeV}$ and no binding for $D D^{*}$ agrees well with some previous calculations [4, 5] in the consituent quark model, since their model parameters reproduce well $\Upsilon, J / \psi$, 
$\Lambda_{b}$ and $\Lambda_{c}$. On the other hand, model calculations which used unrealistically strong pion-exchange interacton yielded very different predictions for the dimesons (many bound states); they were of course irrelevant since they would strongly overbind $\Lambda_{b}$ and $\Lambda_{c}$ too.

Experimental verification of our predictions would be a challenge since it would support the " $V_{Q Q}=\frac{1}{2} V_{Q \bar{Q}}$ rule"

\section{References}

[1] Silvestre-Brac, B., Gignoux, C.: Phys. Rev. D32, 743 (1985); Richard, J.M.: Phys. Rep. 212, 1 (1992)

[2] Silvestre-Brac, B.: Few-Body Systems 20, 1 (1996)

[3] Glozman, L.Ya., Papp, Z., Plessas, W.: Phys. Lett B 381, 311 (1996)

[4] Silvestre-Brac, B., Semay, C.: Z. Phys. C57, 273 (1993); C59, 457 (1993)

[5] Brink D. M., Stancu, Fl.: Phys. Rev. D57, 6778 (1998)

[6] Janc, D.: Diploma Thesis, University of Ljubljana, Ljubljana 1999

[7] Bhaduri, R.K., Cohler, L.E., Nogami, Y.: Nuovo Cim. A65,376 (1981)

[8] Lichtenberg, D.B., Roncaglia, R., Predazzi, E.: J. Phys. G 23, 865 (1997)

[9] Quigg, C., Rosner, J.L.: Phys. Lett B71, 153 (1977); Phys. Reports 56, 167 (1979)

\section{A Input data and model parameters}

In order to be unambiguous we list in table 2 the baryon and meson masses which we use as input for our phenomenological estimate.

We also list in tables 3 and 1 the model parameters of Bhaduri et al. [1] and of Silvestre-Brac and Semay [2] which we need in Sect.3 for our refinements. To unify the notation we write down the form of the effective quark-quark interaction 


$$
\begin{aligned}
V & =-\frac{\overrightarrow{\lambda_{i}}}{2} \cdot \frac{\overrightarrow{\lambda_{j}}}{2}\left\{-\frac{\alpha_{s}}{r}+\kappa+\lambda r+\frac{\tilde{\alpha}_{s} 2 \pi(\hbar c)^{3}}{3 m_{i} m_{j} c^{4}} \sigma_{i} \cdot \sigma_{j} \delta^{3}(\vec{r})\right\} \\
\delta^{3}(\vec{r}) & \rightarrow \frac{\exp \left(-r / r_{0}\right)}{4 \pi r r_{0}^{2}} \text { or } \quad \frac{\exp \left(-r^{2} / r_{0}^{\prime 2}\right)}{\left(\sqrt{\pi} r_{0}^{\prime}\right)^{3}}, \quad r_{0}^{\prime}=A\left(\frac{2 m_{i} m_{j}}{m_{i}+m_{j}}\right)^{-\nu} .
\end{aligned}
$$


Table 1: Seven configurations for $T_{b b}$ with $I S P=10^{+}$. The spatial wave function of $T_{b b}$ is constructed from two delta functions for the two heavy quarks and two Gaussians for the two light quarks. The lower index \pm characterises the permutational symmetry of two identical particles.

$$
\begin{aligned}
\left.\begin{array}{l}
M(b) \\
N(b)
\end{array}\right\}=\delta\left(\mathbf{r}_{b} \pm \mathbf{r} / 2\right), & \left.\begin{array}{c}
m(b) \\
(b)
\end{array}\right\}=\left(\frac{1}{\sqrt{\pi} k}\right)^{-\frac{3}{2}} \exp \left(-\frac{\left(\mathbf{r}_{q} \pm \mathbf{r} / 2\right)^{2}}{2 k^{2}}\right) . \\
\phi_{1+} & =\frac{1}{\sqrt{2}}\left[m\left(q_{1}\right) m\left(q_{2}\right)+n\left(q_{1}\right) n\left(q_{2}\right)\right] \\
\phi_{2+} & =\frac{1}{\sqrt{2}}\left[m\left(q_{1}\right) m\left(q_{2}\right)-n\left(q_{1}\right) n\left(q_{2}\right)\right] \\
\phi_{3 \pm} & =\frac{1}{\sqrt{2}}\left[m\left(q_{1}\right) n\left(q_{2}\right) \pm n\left(q_{1}\right) m\left(q_{2}\right)\right] \\
\psi_{ \pm} & =\frac{1}{\sqrt{2}}\left[M\left(b_{1}\right) N\left(b_{2}\right) \pm N\left(b_{1}\right) M\left(b_{2}\right)\right]
\end{aligned}
$$

\begin{tabular}{lllllll}
\hline & \multicolumn{3}{c}{$q q$} & & $b b$ \\
& $R_{q_{1}, q_{2}}$ & $S$ & $C$ & $R_{b_{1}, b_{2}}$ & $S$ & $C$ \\
\hline 1 & $\phi_{3+}$ & 0 & $\overline{3}$ & $\psi_{+}$ & 1 & 3 \\
2 & $\phi_{1+}$ & 0 & $\overline{3}$ & $\psi_{+}$ & 1 & 3 \\
3 & $\phi_{3-}$ & 1 & $\overline{3}$ & $\psi_{-}$ & 0 & 3 \\
4 & $\phi_{3+}$ & 1 & 6 & $\psi_{+}$ & 0 & $\overline{6}$ \\
5 & $\phi_{1+}$ & 1 & 6 & $\psi_{+}$ & 0 & $\overline{6}$ \\
6 & $\phi_{2+}$ & 1 & 6 & $\psi_{-}$ & 1 & $\overline{6}$ \\
7 & $\phi_{3-}$ & 0 & 6 & $\psi_{-}$ & 1 & $\overline{6}$ \\
\hline
\end{tabular}


Table 2: Masses of some baryons and mesons in MeV. Third and sixth columns give the hyperfine average of the vector meson and the pseudoscalar meson above it

\begin{tabular}{llllll}
\hline$\Lambda_{b}$ & 5624 & & $\Lambda_{c}$ & 2284.9 & \\
& & & $\eta_{c}$ & 2979.8 & \\
$\Upsilon$ & 9460.4 & & $J / \psi$ & 3096.9 & 3067.6 \\
$B_{s}$ & 5369 & & $D_{s}$ & 1969 & \\
$B_{s}^{*}$ & 5416 & 5404 & $D_{s}^{*}$ & 2112 & 2076 \\
$B$ & 5279 & & $D$ & 1867 & \\
$B^{*}$ & 5325 & 5314 & $D^{*}$ & 2008 & 1973 \\
$K$ & 495.7 & & & & \\
$K^{*}$ & 893.9 & 794.3 & & & \\
\hline
\end{tabular}

Table 3: Choice of quark masses

\begin{tabular}{llrr}
\hline Author & & Bhaduri & $\begin{array}{r}\text { Silvestre- } \\
\text {-Brac }\end{array}$ \\
\hline$m_{u, d} \equiv q$ & $\mathrm{MeV}$ & 337 & 315 \\
$m_{s} \equiv s$ & $\mathrm{MeV}$ & 600 & 577 \\
$m_{c} \equiv c$ & $\mathrm{MeV}$ & 1870 & 1836 \\
$m_{b} \equiv b$ & $\mathrm{MeV}$ & 5259 & 5227 \\
\hline
\end{tabular}

Table 4: Choice of parameters for the quark-quark interaction

\begin{tabular}{llrr}
\hline Author & & $\begin{array}{r}\text { Bhaduri } \\
(B D)\end{array}$ & $\begin{array}{r}\text { Silvestre- } \\
\text {-Brac }\end{array}$ \\
& & $A L 1)$ \\
\hline$\alpha_{s}$ & $\mathrm{MeV} \mathrm{fm}$ & 77.0 & 75.0 \\
$\kappa$ & $\mathrm{MeV}$ & -685.1 & -624.1 \\
$\lambda$ & $\mathrm{MeV} / \mathrm{fm}$ & 705.7 & 628.4 \\
$\tilde{\alpha}_{s}$ & $\mathrm{MeV} \mathrm{fm}$ & 462.0 & 257,37 \\
$r_{0}$ & $\mathrm{fm}$ & 0.4545 & \\
$A$ & $\mathrm{GeV}^{\nu-1}$ & & 1.6553 \\
$\nu$ & & & 0.2204 \\
\hline
\end{tabular}

